\title{
Medicina y artes: un curso interdisciplinario usando el aprendizaje basado en problemas en el Grado de Medicina
}

Lilith Lee

Introducción. Este artículo describe una optativa interdisciplinaria, 'Disease and Medicine in the Plastic Arts', ofrecida por la Facultad de Medicina de la Universitat de Girona, incluyendo los resultados y reflexiones sobre esta experiencia.

Materiales y métodos. A través de la observación y descripción de tres cuadros de arte, los estudiantes trabajaron sobre tres diferentes temas médicos. La estrategia de aprendizaje utilizada fue el aprendizaje basado en problemas (ABP). Además, se incluyeron un seminario impartido por una historiadora de arte y dos ejercicios escritos sobre fotografías.

Resultados. Veintidós estudiantes se matricularon en la optativa, divididos en dos grupos. El examen de ABP y los ejercicios escritos que realizaron mostraron su progreso.

Conclusiones. Los comentarios de los estudiantes en las encuestas son positivos, aunque el número de encuestas completadas no es alto, lo que constituye una tarea a mejorar en el futuro. Treinta y seis estudiantes han elegido esta optativa como su preferencia para el próximo curso (2012-2013), lo cual es indicativo del éxito de esta iniciativa y del progresivo interés de los estudiantes sobre la integración de la medicina y las humanidades.

Palabras clave. Aprendizaje basado en problemas. Arte. Educación médica. Estudios interdisciplinarios. Humanidades. Medicina en arte.

Medicine and arts: an interdisciplinary course using problem-based learning in the medical programme

Introduction. This article describes an interdisciplinary elective, 'Disease and Medicine in the Plastic Arts', offered by the Medical School at the University of Girona, including the results and reflections of this experience.

Materials and methods. Through the observation and description of three paintings, the students worked on three different medical issues. The learning strategy was problem-based learning (PBL). Furthermore, there was a seminar given by an art historian and two write-ups on photographs.

Results. Twenty-two students registered for the elective, from which two groups were formed. The PBL exam and writeups showed progress of the students.

Conclusions. The comments of the students in the survey are positive, although the number of students that has responded is not very high, which is a task for improvement in the future. Thirty-six students have chosen this elective as their preference for the following academic year (2012-2013), which shows the success of this initiative and the rising interest of the students in the integration of medicine and humanities.

Key words. Art. Humanities. Interdisciplinary studies. Medical education. Medicine in art. Problem-based learning.

\section{Introducción}

En el plan de estudios de la Facultad de Medicina de la Universitat de Girona se incluyen 25 créditos ECTS para las optativas, que permiten la introducción de temas específicos relacionados con el estudio de medicina o materias interdisciplinarias que no se contemplan en la parte troncal del programa. Estas optativas se ofrecen a los estudiantes desde el segundo curso hasta el sexto. Para fomentar el de- sarrollo de la competencia 38 de la memoria del Grado de Medicina de la facultad, 'Garantizar el conocimiento adecuado de la lengua inglesa tanto oral como escrita teniendo en cuenta las especificidades del registro médico para poder comunicarse eficazmente en la comunidad internacional científica y profesional,' se imparten completamente en inglés, es decir, esta lengua se usa en todas las actividades y los materiales utilizados. Otra característica de las optativas es la mezcla de estudiantes de
Facultad de Medicina. Universitat de Girona. Girona, España.

Correspondencia: Dra. Lilith Lee. Facultad de Medicina. Universitat de Girona. Emili Grahit, 77. E-17071 Girona.

Fax: +34972419617.

E-mail: lilith.lee@udg.edu

Agradecimientos: Luis Branda, por su apoyo y consejos en distintas facetas de la implementación de la optativa y la lectura del manuscrito.

Conflicto de intereses: No declarado.

Competing interests: None declared.

(c) 2014 FEM 
Tabla I. Objetivos de aprendizaje.

In this module the students are expected to:

Develop skills of observation and interpretation

Describe how artists portray manifestation of health-related issues

Communicate and express what has been observed

Relate health issues to their social and historical contexts

Transfer the skills learnt from appreciation of artworks to patient care

distintos cursos, lo que facilita el intercambio de ideas y experiencias.

La estrategia educacional dominante es el aprendizaje basado en problemas (ABP). El número de problemas o situaciones que se utiliza depende de la duración de cada módulo. Para las optativas con una duración de tres semanas se espera que el estudiante explore tres problemas. Cada problema supone tres sesiones de dos horas de duración cada una. En la primera, el grupo, a partir de un problema, identifica una lista de objetivos de aprendizaje a estudiar. En las dos sesiones posteriores, el grupo comparte la información buscada por sus miembros y discute lo que ha estudiado para cumplir los objetivos. Debido a la limitación de los recursos de la facultad, el tamaño de los grupos es de 10-11 alumnos y cada grupo cuenta con un tutor. El desempeño del estudiante en las tutorías de ABP se evalúa con una rúbrica - diseñada a partir de los trabajos realizados en la Universidad Nacional del Sur (Argentina), modificada posteriormente en la Escuela Universitaria de Enfermería de Vall d'Hebron (Barcelona) y adaptada al Grado de Medicina y el diseño curricular de la facultad en la Universitat de Girona- que examina cuatro áreas en las cuales el estudiante debe demostrar estas competencias: habilidades de aprendizaje, comunicación, responsabilidad y relaciones interpersonales. Cada una de estas áreas es ponderada de la misma manera y corresponde al $25 \%$ de la nota del desempeño del estudiante en el ABP. Cada una de las áreas consta de cinco ítems que definen los distintos criterios utilizados en la evaluación. La evaluación basada en esta rúbrica corresponde al menos a un $40 \%$ de la nota final que el estudiante recibe en la optativa. En la mitad de la optativa y en la última tutoría, el grupo, incluyendo el tutor, hace una evaluación oral que contiene tres partes: autoevaluación, evaluación por pares (coevaluación) y evaluación por el tutor. El tutor del grupo es la persona que cumplimenta la rúbrica que se enviará al coordinador de la optativa.
El examen principal es un ejercicio de evaluación basado en problemas, conocido como 'examen ABP'. El examen ABP está desarrollado por Luis Branda, el actual director de la Unidad de Educación Médica de la facultad. Las etapas se basan en el Problem-based Learning Evaluation Exercise (PBEE) que se desarrolló en la Facultad de Ciencias de la Salud de la McMaster University canadiense [1,2]. El examen ABP de la optativa que se describe en este artículo difiere un poco, de acuerdo con los objetivos del curso. Consta de dos partes. La primera parte consiste en uno o dos problemas, sobre los cuales los estudiantes establecen hipótesis sobre lo observado e identifican los objetivos relevantes a estudiar. Se dispone de un período (1-2 días) para estudiar y los estudiantes regresan para realizar la segunda parte, en la cual contestan preguntas que corresponden con lo que cada uno ha indicado como plan de trabajo en la primera parte. Esta parte de la evaluación -el examen ABP-corresponde al menos al $20 \%$ de la nota final de la optativa.

La optativa 'Disease and Medicine in the Plastic Arts' (en adelante, M\&A) sigue estrictamente estos criterios establecidos por la Unidad de Educación Médica de la facultad y la descripción del plan de estudios del Grado de Medicina. M\&A se ofreció la primera vez en el curso académico 2011-2012. El diseño de la optativa se centra en el desarrollo de varias habilidades que tienen en común la medicina y la apreciación del arte: las habilidades de observación, descripción, interpretación y comunicación de lo que se ha observado (Tabla I).

\section{Materiales y métodos}

En la M\&A, aparte de las tutorías de ABP, se llevaron a cabo otras actividades: un seminario y dos ejercicios por escrito. En los siguientes apartados, se describe el contenido de cada actividad.

\section{Tutorías de ABP}

Para la optativa M\&A, los problemas se presentan en forma de reproducciones de cuadros de arte en diapositivas. A través de la observación de los cuadros, los estudiantes identifican temas de estructura, función y patología relevante. La selección de cuadros se basa en la posibilidad de fácil identificación de esos temas y su relevancia a los módulos que los estudiantes habían cursado. En concreto, los tres temas elegidos son los que posiblemente los estudiantes hayan conocido pero no estudiado en profundidad, lo que les facilita reconocerlos. De esta 
manera, los estudiantes, usando sus conocimientos previos, tienen la oportunidad de explorar los temas específicos en más profundidad y desde una perspectiva diferente.

Tomando estos elementos en cuenta, se eligieron las siguientes obras de arte como problemas. El primer problema fue el Autorretrato con collar de espinas (1940), de Frida Kahlo (1907-1954) [3], que facilita enfocar el hirsutismo. El segundo problema fue el Opistótonos (1809), de Sir Charles Bell (1774-1842) [4], que trata del tétanos. El tercer problema consistió en cuatro autorretratos de Rembrandt (1606-1669), pintados en 1632, 1640, 1659 y 1669 [5], y seleccionados por la similitud en la postura, la certeza de la autoría y los cambios que se pueden notar en la facciones del pintor. La presentación de este tercer problema fue más elaborada. Antes de ver los autorretratos, a los estudiantes se les mostró un fragmento del vídeo Rembrandt, schilder van de mens (1956), de Bert Haanstra [6], en que el director vincula distintos autorretratos que el artista hizo a lo largo de su vida en una secuencia continuada usando los ojos como eje y recreando para los espectadores el proceso de envejecimiento de Rembrandt.

Además, los tres problemas representaban un retroceso en el tiempo, desde un período comparativamente más familiar a los estudiantes hasta una época anterior.

Desde la observación del cuadro (o de los cuadros, en el caso del tercer problema), los estudiantes describieron los distintos elementos e identificaron los temas relacionados con la medicina que estudiarían. De este modo, los cuadros no sólo sirvieron para desarrollar las habilidades de observación, descripción y comunicación, sino también como catalizadores en la adquisición de conocimientos relacionados con la formación de medicina. Además, para poder apreciar algunos elementos de los cuadros, los estudiantes tuvieron que buscar información sobre la vida y las otras obras de los artistas, y temas relevantes a las distintas épocas. Por ello, se dieron cuenta de la importancia de los contextos sociales e históricos, que es uno de los objetivos de aprendizaje de la optativa.

Durante el curso, se ofreció a los estudiantes la posibilidad de consultar a expertos en cada área que comprendía la optativa, incluyendo la apreciación del arte.

\section{Ejercicios escritos}

Otra actividad involucró fotografías. En la primera sesión de tutoría, además de la exploración del
Tabla II. Guías para los ejercicios escritos.

Write-up 1. Guidelines for areas that you should include:

Describe what you see in the photo

From what you have described from the photo, summarize the situation that is represented in it, such as the time period and people that appear

Make an interpretation/conclusion of what you see

Think about the questions that you would consider asking in order to find out more about the photo/situation

Based on these questions, propose areas for further investigation

Write-up 2. Guidelines for areas that you should include:

Use the skills and knowledge that you have learnt in this module to describe what you see in the photo

From what you have described from the photo, summarize the situation that is represented in it, such as the time period and people that appear

Make an interpretation/conclusion of what you see

problema, se mostró una fotografía del libro $U n$ hombre afortunado, de John Berger y Jean Mohr [7], donde se analiza en profundidad el trabajo que realizó el Dr. Sassall en un pueblo rural de la Inglaterra de los años sesentas, buscando las razones por las cuales a Sassall se le consideraba un buen médico. La fotografía captura el momento de una consulta o visita de Sassall con una mujer, presumiblemente una paciente suya. El ejercicio consistió en que, en media hora, los estudiantes escribieran una descripción de lo que observaron en la foto y sacaran conclusiones. Una serie de preguntas guió a los estudiantes en la tarea (Tabla II). En la mitad de la optativa, los estudiantes realizaron el mismo ejercicio sobre otra fotografía de la misma consulta/visita. El objetivo de esta actividad fue seguir el progreso en la adquisición de las habilidades de observación, descripción e interpretación, y guiarlos con preguntas cómo se puede comenzar a realizar una observación. Desde que cada uno de los estudiantes recibió comentarios detallados sobre lo que había escrito, esta actividad permitió ofrecerles retroalimentación de su desempeño individual.

\section{Seminario}

Se programó un seminario de dos horas, impartido por una historiadora del Centro de Estudios Da- 
linianos, que consistió en una exposición sobre la apreciación del arte y del proceso de investigación histórica de cuadros de arte, usando las obras de Dalí como ejemplo. El objetivo de esta actividad consistió en introducir elementos artísticos e ilustrar una perspectiva distinta, pero a la vez semejante, entre la investigación de historiadores del arte sobre las pinturas y de los médicos sobre las enfermedades de los pacientes: 'Similarities, coincidences and parallelisms when an art historian looks at a painting and a doctor treats a patient. True or false?.

\section{Evaluación}

La evaluación en M\&A consistió en dos partes: la evaluación del desempeño del estudiante en las tutorías de $\mathrm{ABP}(50 \%)$ y el examen ABP (50\%).

\section{Evaluación de las tutorías de ABP}

Aparte de la puntuación recibida en la rúbrica, cada estudiante recibió también comentarios escritos sobre su desempeño en las tutorías.

\section{Examen $A B P$}

El examen ABP es un ejercicio de evaluación basado en problemas, formado por dos partes y consistente en tres etapas; en total, cuatro horas presenciales. En la primera etapa, los estudiantes observaron un cuadro que, para este curso, fue Mi nana $y$ yo (1937), de Frida Kahlo [3], la artista con la que los estudiantes estaban familiarizados a través del primer problema. Además de describir el cuadro -géneros del cuadro, elementos y composición, relación con la vida de la pintora, relación con otras de sus obras, símbolos e interpretaciones-, se requería que los estudiantes identificaran los temas de relevancia médica y propusieran un plan de estudio a seguir. En este caso, los temas de hirsutismo y el reflejo de eyección láctea eran los más evidentes. El examen se realizó de forma individual y sin discusión grupal.

En la segunda etapa, después de la parte primera presencial, los estudiantes tuvieron dos días para buscar información y estudiar los temas del plan de estudio que cada uno se había propuesto. Mientras, un experto asignaba las preguntas sobre temas de acuerdo con el plan de estudio de cada estudiante, es decir, las preguntas eran individualizadas.

La tercera etapa corresponde a la segunda parte del examen. Se distribuyeron las preguntas asignadas a cada estudiante sobre los temas identificados por cada uno de ellos; en esta parte dispusieron de dos horas para responder a las preguntas.

\section{Resultados}

Veintidós estudiantes se matricularon para la optativa, que se dividieron en dos grupos. Cinco eran del segundo curso, y diecisiete, del tercero. Fue la primera optativa que cursaron los estudiantes de segundo; para los de tercero, los estudiantes ya habían cursado previamente otras optativas. Todos los estudiantes tenían experiencia en el ABP.

A cada grupo se le asignó la misma docente tutora, que también fue la coordinadora que había diseñado la optativa. En este sentido, se puede considerar que esto permitió lograr una óptima coherencia entre el diseño y la práctica, y la misma facilitación de las tutorías de los dos grupos.

Con respecto a la recepción de los problemas por los estudiantes, la vida de los artistas les parecía plantear un área de mucho interés. Los estudiantes se relacionaban especialmente con Frida Kahlo y Sir Charles Bell por sus vínculos con la medicina. La vida turbulenta de Frida estimuló al mismo tiempo interés y compasión por su frustrada vocación de estudiar medicina; la de Bell actuó como 'ejemplo a seguir'. Una estudiante comentó al grupo por qué ellos no podían hacer lo mismo, combinar distintas áreas de interés: hacerse artista y médico. Al conocer más la vida de Bell, dos estudiantes incluso lo defendieron con respecto a sus conflictos profesionales. Desde la observación del autorretrato de Frida, aparte del hirsutismo, notaron signos de depresión en su mirada, sin enfoque en un punto particular. Por otra parte, las diferencias en la apariencia entre el joven y el anciano Rembrandt, tanto en la expresión como en las prendas, despertaron curiosidad e incitaron especulaciones hacia su vida, de cómo iba de bien a peor. Intentaron descifrar los significados y hacer hipótesis de lo que habían observado en los rostros desde las vidas registradas de los artistas. De este modo, los estudiantes utilizaron el poder de la observación como catalizador para investigar y recoger información relevante de los cambios con el envejecimiento. La identificación de tétanos en el segundo problema tampoco presentó dificultades. Varios estudiantes reconocieron la enfermedad. Aunque no es frecuente en los países europeos, los estudiantes apreciaron la oportunidad de estudiar la etiología de la enfermedad, la composición de la vacuna y su mecanismo de acción, que les facilitó la posibilidad de explicar su importancia a los pacientes en el futuro.

Ambos grupos se interesaron en buscar y revisar las otras obras de los artistas para construir un contexto del cuadro seleccionado y para identificar los estilos de los artistas a través de los elementos y 
los temas recurrentes. Para el tercer problema con los cuadros de Rembrandt, una estudiante preparó una presentación de diapositivas en orden cronológico con el fin de reforzar la discusión del grupo sobre el envejecimiento.

Con respecto al seminario, se distribuyó un cuestionario con siete preguntas, cuyo resultado fue muy positivo: una media de 9,1 sobre 10 para la evaluación global. Asistieron catorce estudiantes -la asistencia al seminario no era obligatoria- y trece completaron el cuestionario, que se distribuyó en la siguiente tutoría.

Además, se llevó a cabo una evaluación continuada. Tras finalizar el primer problema, la tutora hizo una evaluación individual de cada estudiante dentro de la tutoría, dándoles retroalimentación de su participación y rendimiento hasta aquel momento. Dependiendo de la necesidad del grupo, la retroalimentación podría ser más frecuente. Por ejemplo, en este curso, uno de los grupos necesitaba más seguimiento y se lo proporcionó la tutora después de cada tutoría; en algunos casos, con más detalle, y en otros, más resumido.

Por otra parte, la universidad dispone de una encuesta general para los módulos y las asignaturas de todas las facultades, abierta a los estudiantes al terminar cada módulo o asignatura. Sólo seis estudiantes cumplimentaron la encuesta. Esta falta de cumplimiento en completar las encuestas, que se observa en todos los módulos de medicina, ha constituido un problema serio para la facultad.

La media de la evaluación global fue de 8,7 sobre 10. Hubieron dos comentarios, ambos sobre los objetivos: uno, que solicitó objetivos más claros, y el otro, que agradeció a la coordinadora el diseño y ayuda para cumplir los objetivos. Posteriormente, se envió a los estudiantes un cuestionario especialmente diseñado para la optativa, con el propósito de investigar el impacto en el aprendizaje y el desarrollo personal de los estudiantes de la optativa. Cinco respondieron y los comentarios fueron positivos.

Todos los estudiantes, salvo una, superaron la evaluación de tutorías de ABP. La media fue de 78 sobre 100, y todos aprobaron el examen con una media de 79 sobre 100 .

\section{Discusión}

La incorporación de las artes en la educación médica encontró sus promotores en los años noventa en Estados Unidos con la creencia de que, al exponerse a ellas, los estudiantes tomarían conciencia de la importancia de una observación cuidadosa en una situación clínica [8-15]. Berger [16], en 1980, señaló la importancia de fomentar las habilidades de observación clínica de los médicos en formación y apuntó cuatro niveles que van más allá de descartar enfermedades posibles. Luego, Boudreau et al [17] agregaron uno más al diseñar un curso de observación clínica basado, entre otros, en los principios de la apreciación del arte en la McGill University. Después de la publicación del documento Tomorrow's Doctors (1993), se han descrito cursos de arte introducidos en las facultades de medicina del Reino Unido [18,19]. En España, algunas facultades de medicina también han empezado a seguir este enfoque -como se ha registrado en sus planes de estudios-, introduciendo optativas de artes visuales, como una combinación de cine, literatura y medicina. Esto se ha visto en la Universidad de Oviedo, en la Universitat Autònoma de Barcelona y en la Universidad Complutense de Madrid, la cual también ofrece otra optativa, 'Enfermedad y medicina en el arte I y II'. Existen además intentos aislados que usan una película como catalizador para la discusión de temas específicos [20].

La implementación, el diseño y la magnitud de estos intentos varían desde una colaboración entre facultades de medicina y expertos de otras áreas y los museos locales $[8,10,13-15,17]$ a empeños individuales $[9,18]$. Varían en extensión de unas tres sesiones [10] a una continuada de ocho meses [12], de visitas al museo $[8,10,12,14,15]$ y el uso de cuadros o fotografías o una mezcla de ambos [8-10,12$14,17,18]$ a danzas y teatro [13,18,19]. Forman parte del plan de estudios [9,12,17-19] o son actividades extracurriculares $[13,19]$. Incluyen un análisis cualitativo y cuantitativo y comparativo [12, 14,19] o sólo comentarios de los participantes [9, $10,15,17,18]$. Todos estos cursos tienen como objetivo mejorar la habilidad de observación de los estudiantes a través de la apreciación del arte, y que ellos sean conscientes de transferir esta habilidad al diagnosticar. Algunos subrayan la empatía que los estudiantes podrían desarrollar a través de la apreciación del arte, que contribuiría al cuidado de los pacientes. Otros pretenden ofrecer un espacio de reflexión y relajación para los estudiantes, la importancia del cual Gordon [21] ha presentado sucintamente.

En la Facultad de Medicina de la Universitat de Girona se ha aprovechado la flexibilidad de las optativas con el fin de crear un espacio para un curso sobre la medicina y las artes. Este constituye el primer intento de introducir el estudio de temas interdisciplinarios que incluyen las humanidades en la facultad. 


\section{Puntos para la reflexión}

\section{Diferencias entre los dos grupos}

Como se ha mencionado, había dos grupos en la optativa. Ambos grupos (A1 y A2) disponían no sólo de los mismos materiales, sino también de la misma tutora. Sin embargo, con un mínimo margen, la dinámica entre los dos grupos se diferencia de manera importante. Al principio, los dos grupos necesitaron un período de adaptación por la naturaleza y el contenido de la optativa, por su diferencia con el resto de los módulos del Grado de Medicina. Con el primer problema, los estudiantes se familiarizaron con los objetivos y las expectativas de la optativa. El grupo A1 rápidamente estableció un modus operandi para abordar los problemas y un orden interno para identificar y cumplir los objetivos. En la sesión de la evaluación, muchos estudiantes mencionaron este proceso, que coincide con la observación de la tutora. En cambio, al grupo A2 le costó mucho decidir los temas o los objetivos a discutir y estudiar, y no logró tener un ritmo adecuado hasta que llegaron al último problema.

La participación y la discusión grupal no plantean las únicas diferencias entre los dos grupos. A ambos se les había ofrecido la posibilidad de consultar libros de textos electrónicos que la biblioteca no tiene, a través del acceso personal que posee la coordinadora. Su uso dependía de cada estudiante, que debía hacer el esfuerzo de buscar los capítulos relevantes y escribir un mensaje a la coordinadora solicitando el material. Varios estudiantes del grupo A1 solicitaron este recurso de forma repetida durante toda la optativa, y más estudiantes lo aprovecharon al acercarse la fecha del examen. Por lo contrario, ningún miembro del grupo A2 lo solicitó. Por otra parte, salvo dos estudiantes del grupo A1, todos asistieron al seminario - cuya asistencia no era obligatoria-, mientras que sólo asistieron cinco de los once estudiantes del grupo A2.

Podría ser razonable concluir que las diferencias notadas se deben a las actitudes de aprendizaje. La rúbrica de la evaluación de las tutorías de $\mathrm{ABP}$ subraya la importancia de este aspecto. En las tutorías, la tutora tenía la oportunidad de observar y seguir el progreso de cada estudiante durante toda la optativa - la tutora tomó notas detalladas de la discusión en las tutorías, que formó la base para la evaluación de las tutorías de ABP. Después de cada tutoría, la tutora organizó las notas para seguir el progreso de cada estudiante. Dos horas de tutorías suponen cuatro horas para organizar las notas. Al final del módulo, la tutora tenía un seguimiento íntimo del proceso de aprendizaje de cada estudiante-, lo que le permitió estimar, además de las habilidades de aprender el contenido, el comportamiento y las actitudes. En el grupo A1 sólo una estudiante mostró falta de interés de manera continuada, mientras que en el grupo A2, siete estudiantes no mostraron un interés continuado.

Resulta razonable preguntarse si parte de la explicación de la falta de motivación es que los estudiantes no pudieron elegir cursar la optativa de su preferencia. A los estudiantes se les solicitó que indicaran sus preferencias entre las seis optativas ofrecidas. Los que fueron asignados a la M\&A la habían elegido en la primera opción, o en la segunda si su primera opción ya estaba completa.

Las diferencias entre los grupos de ABP presentan cuestiones intrigantes. Aunque la implementación, la cultura y los estudios sean diferentes, quizá puedan aprovecharse las observaciones de algunos estudios. La importancia de conocer los fines y los objetivos del ABP es uno de los factores que contribuyen a un funcionamiento efectivo del grupo [22]. El grupo A2 encontró muchas dificultades en entender los objetivos de la optativa, que podría ser una razón determinante de la ineficacia del grupo, aunque no explica la diferencia entre ambos grupos. En algunas publicaciones se propone dividir a los estudiantes en grupos homogéneos como una posible solución a la variación observada, aunque también esto conlleva otros problemas [23]. Kinnunen y Malmi [24] resumen factores que contribuyen a grupos efectivos e inefectivos. Además subrayan que algunos necesitan más tiempo para saber cómo trabajar juntos y, en algunos grupos, la falta de participación de un número alto de miembros es un detrimento.

Los distintos factores y las sugerencias de estos estudios en cómo mejorar el rendimiento de los grupos de ABP se tomarán en cuenta para diseñar estrategias factibles para el curso próximo.

\section{Actitud del aprendizaje: medicina frente a artes}

La particularidad de la optativa -que no trata de una única manera los temas médicos- ofrece una oportunidad para reflexionar cómo la actitud afectaría el proceso de aprendizaje y la adquisición de habilidades críticas. Se cita un ejemplo para ilustrar el argumento. Al buscar información sobre la vida de Rembrandt, se encontraron discrepancias entre los datos recogidos por distintos estudiantes. Al final, los estudiantes decidieron que no era necesario resolverlas porque no las consideraron importantes. Al ser desafiados por la tutora sobre cómo actuarían si la discrepancia se produjera en el área médica, respondieron que buscarían más libros 
para encontrar la respuesta, pero no vieron la necesidad de realizar este proceso porque aprender más sobre la vida de Rembrandt no les serviría en su carrera de medicina. En este caso se podría concluir que los estudiantes poseen la habilidad y conocen el procedimiento de investigación y verificación de la validez de la información. Sin embargo, cuando se enfrentaron con las contradicciones en la información sobre el período de un tratamiento para hirsutismo, tampoco trataron de resolver la discrepancia o hallar más información sobre las distintas condiciones, y lo dejaron sin resolver. Por tanto, no resulta tan fácil separar las áreas 'importantes para medicina' de las que no lo son para aplicar el pensamiento crítico.

\section{Análisis de los comentarios de los estudiantes}

Para poder evaluar la optativa y averiguar si ha cumplido los objetivos que se intentaban cumplir, se distribuyó a los estudiantes un cuestionario después de terminar el módulo (Tabla III). Aunque se dispone de una encuesta de la universidad para evaluar las asignaturas, las preguntas son muy genéricas y difíciles de ajustar a las particularidades de una optativa implementada con el ABP con el objetivo de desarrollar las habilidades transversales de los estudiantes.

Los cinco estudiantes que respondieron explicaron que la optativa los atrajo por la idea de combinar la medicina y las artes, y dos de ellos pensaban que la optativa tenía alguna relación con la historia de la medicina cuando la eligieron. Con respecto al desarrollo personal, mencionaron que les había ayudado a mejorar las habilidades de descripción, observación y comunicación. Además, aprendieron que existían numerosos puntos de vista y que era importante saber valorar opiniones, lo cual los ayudó a fomentar la reflexión. Reconocieron la importancia de los detalles. Se dieron cuenta de que los cuadros tenían sus propias historias, a través de las cuales era posible aprender lo científico, y cómo la vida de los artistas afectaba su producción artística. También mencionaron que habían ganado conocimientos sobre los tres artistas y habían mejorado su expresión en inglés. Con respecto a la relevancia de la optativa en el Grado de Medicina, reconocieron que los ayudó a ser más conscientes y sensibles a lo que se puede observar con los gestos de los pacientes y la importancia de sus individualidades, especialmente las distintas expresiones de dolor. Tuvieron la oportunidad de entender la medicina desde otros puntos de vista. Tres respondieron que les interesaría tener otra ocasión de escoger un módulo similar. Todos recomendarían la optativa a otros estudiantes.
Tabla III. Cuestionario sobre la optativa.

What are the reasons that led you to choose this elective?

Does the content of this elective contribute to your personal development (for example, broadening your education, widening your viewpoints, developing transferable skills, sharpening your observation, fostering self-reflection)? If yes, in what way?

Do you think the content and skills that you learnt in this elective have helped or will help you in other parts of the medical programme? If yes, in what way?

Would you recommend this elective to other students?

Overall, how would you rate this elective? (1 not satisfied - 7 very satisfied)

Other comments

Para estos estudiantes, la optativa logró ampliar sus perspectivas e introducir otros puntos de vista que los ayudan a desarrollar habilidades transversales, de las cuales consideraron que contribuirían al cuidado de los pacientes.

Las respuestas corresponden a un número muy limitado; sin embargo, para estos estudiantes la experiencia supone un beneficio. Una tarea por hacer para próximos cursos es concienciar a los estudiantes de la importancia de responder al cuestionario para una adecuada evaluación del programa. Para el próximo curso, 36 estudiantes han escogido esta optativa como su primera opción, y otros 24 han escogido como primera preferencia una optativa nueva, 'Medicine in literature', lo cual denota cierto interés y la curiosidad creciente de la población estudiantil hacia estudios interdisciplinarios de la medicina y las humanidades.

En conclusión, con el incremento de cursos que introducen la combinación de humanidades y medicina y los informes positivos de los docentes e investigadores, se ha cuestionado si estos resultados se basan en la evidencia $[25,26]$, particularmente teniendo en cuenta la limitación de los recursos. La respuesta es que aún hay mucho por hacer; pero ya se han realizado algunos estudios cualitativos y estadísticos muy cuidadosos [13,19]. Los investigadores están de acuerdo en que se requiere la búsqueda de instrumentos aptos para poder evaluar los resultados [26-28] y se espera poder analizar los datos sobre los efectos que tendrán estos programas a largo plazo.

Con respecto a esta optativa, la mayoría de los estudiantes han cumplido los objetivos, salvo el 'Transfer the skills learnt from appreciation of art- 
works to patient care, ya que se tiene que esperar a evaluar el desempeño de los estudiantes en la clínica para poder saberlo. La duración de la optativa es muy corta, pero esta limitación se podría superar introduciendo un segundo curso para profundizar el aprendizaje. Para poder evaluar la eficacia de la optativa sería importante recoger las opiniones de los estudiantes de forma más efectiva. Los ejercicios escritos ofrecieron un instrumento útil para observar el progreso de los estudiantes; en el segundo ejercicio, muchos de ellos habían descrito más detalles y habían mejorado en fundamentar sus interpretaciones con evidencias. El número limitado de estudiantes -22 en este curso- hizo que la falta de recursos adicionales no fuera insuperable, pero con un incremento del número de estudiantes puede presentarse un problema. Además, como el curso se imparte completamente en inglés, supone una necesidad de recursos humanos y bibliográficos distinta a los módulos troncales. El apoyo en estas dos áreas sería muy importante para poder llevar a cabo las futuras ediciones con un incremento de número de participantes y la realización de un análisis riguroso.

\section{Bibliografía}

1. Branda LA. Problem-based evaluation exercise, PBEE. In Woods DR, ed. Problem-based learning: resources to gain the most from PBL. Hamilton: Woods; 1999. Chapter F.

2. Woods DR. Preparing for PBL. Hamilton: McMaster University; 2003.

3. Herrera H. Frida Kahlo. The paintings. London: Bloomsbury; 1991.

4. Crumplin MKH, Starling P. A surgical artist at war. The paintings and sketches of Sir Charles Bell 1809-1815. Edinburgh: The Royal College of Surgeons of Edinburgh; 2005

5. White C, Buvelot Q, eds. Rembrandt by himself. London National Gallery Publications; 1990.

6. Haanstra B. Rembrandt: pintor del hombre [VHS]. Amsterdam: Cor Koppies Video.

7. Berger J, Mohr J. A fortunate man. The story of a country doctor. New York: Vintage Books; 1997.

8. Dolev IC, Friedlaender LK, Braverman IM. Use of fine art to enhance visual diagnostic skills. JAMA 2001; 286: 1020-1.

9. Boisaubin EV, Winkler MG. Seeing patients and life contexts: the visual arts in medical education. Am J Med Sci 2000; 319: $292-6$
10. Bardes CL, Gillers D, Herman AE. Learning to look: developing clinical observational skills at an art museum. Med Educ 2001; 35: 1157-61.

11. Rodenhauser P, Strickland MA, Gambala CT. Arts-related activities across U.S. medical schools: a follow-up study. Teach Learn Med 2004; 16: 233-9.

12. Elder NC, Tobias B, Lucero-Criswell A, Goldenhar L. The art of observation: impact of a family medicine and art museum partnership on student education. Fam Med 2006; 38: 393-8.

13. Shapiro J, Rucker L, Beck J. Training the clinical eye and mind: using the arts to develop medical students' observational and pattern recognition skills. Med Educ 2006; 40: 263-8.

14. Naghshineh S, Hafler JP, Miller AR, Blanco MA, Lipsitz SR, Dubroff RP, et al. Formal art observation training improves medical students' visual diagnostic skills. J Gen Intern Med 2008; 23: 991-7.

15. Schaff PB, Isken $S$, Tager RM. From contemporary art to core clinical skills: observation, interpretation, and meaning-making in a complex environment. Acad Med 2011; 86: 1272-6.

16. Berger LR. A hierarchy of clinical observations. Pediatrics 1980; 65: 357-8.

17. Boudreau JD, Cassell EJ, Fuks A. Preparing medical students to become skilled at clinical observation. Med Teach 2008; 30: 857-62.

18. Weller $\mathrm{K}$. The visualization/modification of the body in art and medicine. Int J Healing Caring 2002; 2: 1-11.

19. De la Croix A, Rose C, Wildig E, Willson S. Arts-based learning in medical education: the students' perspective. Med Educ 2011; 45: 1090-100.

20. Aramburu J, Bosch F, Sentí M, Baños JE. Utilidad de las películas para debatir temas complejos: política, religión y ciencia en Ágora. Educ Med 2012; 15: 95-101.

21. Gordon JJ. Medical humanities: state of the heart. Med Educ 2008; 42: 333-7.

22. Mpofu DIS, Das M, Stewart T, Dunn E, Schmidt H. Perceptions of group dynamics in problem-based learning sessions: a time to reflect on group issues. Med Teach 1998; 20: 421-7.

23. Stausholm NT, Topsøe VC. Effects of homogeneous PBL groups [Internet]. Copenhagen: The Multidisciplinary University College of Copenhagen, Institute of Biomedical Technical Science. URL: https://www.ucviden.dk/portal/ files/9472428/PBLgroups.pdf. [24.08.2012].

24. Kinnunen P, Malmi L. Do students work efficiently in a group? Problem-based learning groups in basic programming course. In Korhonen A, Malmi L, eds. Proceedings of the Fourth Finnish/Baltic Sea Conference on Computer Science Education; 2004 oct 1-3; Koli, Finland. Helsinki: Helsinki University of Technology; 2004. p. 57-67.

25. Ousager J, Johannessen $\mathrm{H}$. Humanities in undergraduate medical education: a literature review. Acad Med 2010; 85: 988-98.

26. Perry M, Maffulli N, Willson S, Morrissey D. The effectiveness of arts-based interventions in medical education: a literature review. Med Educ 2011; 45: 141-8.

27. Charon R. Calculating the contributions of humanities to medical practice -motives, methods, and metrics. Acad Med 2010; 85: 935-7.

28. Belling C. Sharper instruments: on defending the humanities in undergraduate medical education. Acad Med 2010; 85: 938-40. 